\title{
Progressive Ankylosis (Ank) Protein Is Expressed by Neurons and Ank Immunohistochemical Reactivity Is Increased by Limbic Seizures
}

\author{
Manuel Yepes, Elizabeth Moore, Sharron A. N. Brown, Heather N. Hanscom, \\ Elizabeth P. Smith, Daniel A. Lawrence, and Jeffrey A. Winkles
}

Departments of Vascular Biology (MY, EM, SANB, HNH, DAL, JAW) and Experimental Pathology (EPS), Jerome $H$. Holland Laboratory for the Biomedical Sciences, American Red Cross, Rockville, Maryland; and Department of Neurology (MY), Georgetown University Hospital, and the Department of Biochemistry and Molecular Biology (DAL, JAW), George Washington University Medical Center, Washington, DC

\begin{abstract}
SUMMARY: Ank is a 492-amino acid multipass transmembrane protein involved in the regulation of extracellular inorganic pyrophosphate levels and the control of tissue calcification. Previous Northern blot hybridization experiments revealed that Ank mRNA was expressed in the brain, but there have been no reports describing the anatomical sites or specific cell types in the brain that express Ank protein. In this study, we demonstrate that Ank is expressed primarily in human brain neurons, with the highest levels of expression observed in the thalamus, the III and V cortical layers, the Purkinje cells of the cerebellum, clusters of cells in the dorsal portion of the pons and midbrain, and neurons of the anterior horn of the spinal cord. In primary mouse neuronal cell cultures, Ank is detected on both the cell body and on cell extensions, mainly dendrites. In the rat brain, Ank mRNA is expressed at relatively high levels in the thalamus, midbrain, and spinal cord, and the Ank protein expression pattern is similar to that observed in the human brain. Finally, we observed a significant increase in Ank immunoreactivity in the rat amygdala, the CA-2 and CA-3 layers of the hippocampus, and the cerebral cortex after the induction of seizure activity. Ank regulation of ATP and/or inorganic pyrophosphate release from neurons may function to modulate the membrane excitability and cell death associated with seizure activity. (Lab Invest 2003, 83:1025-1032).
\end{abstract}

$A$ $\mathrm{nk}$ is a growth factor-regulated gene encoding an $\sim 54-\mathrm{kDa}$ protein with 10 to 12 transmembrane domains, three potential glycosylation sites, and multiple putative phosphorylation sites (Guo et al, 2002; Ho et al, 2000; Nurnberg et al, 2001; Sohn et al, 2002). An autosomal recessive mutation at the Ank locus is the molecular basis for murine progressive ankylosis, a disease characterized by excessive mineralization of articular cartilage, bony outgrowths, and joint destruction (Ho et al, 2000). Ank/ank mutant mice also display a marked increase in cementum formation during tooth development (Nociti et al, 2002). Furthermore, it has been reported that mutations in the human Ank gene can cause autosomal dominant craniometaphyseal dysplasia (Nurnberg et al, 2001; Reichenberger et al, 2001) and some forms of chondrocalcinosis (Pendleton et al, 2002).

\section{DOI: 10.1097/01.LAB.0000075640.49586.E6}

Received February 20, 2003.

This work was supported in part by National Institutes of Health grants NS-02223 (MY), HL-55374 (DAL), HL-55747 (DAL), HL-39727 (JAW), and HL-67051 (JAW).

Address reprint requests to: Dr. J. A. Winkles, Department of Vascular Biology, Holland Laboratory, American Red Cross, 15601 Crabbs Branch Way, Rockville, Maryland 20855.E-mail:winkles@usa.redcross.org
Cell culture studies using either Ank cDNAtransfected COS cells or fibroblasts isolated from wild-type or ank/ank mice have demonstrated that the Ank protein is involved in controlling the intracellular versus extracellular distribution of inorganic pyrophosphate (PPi) (Ho et al, 2000; Pendleton et al, 2002). This compound is generated within cells as a major byproduct of numerous biochemical and biosynthetic reactions (Russell, 1976); furthermore, it can be formed extracellularly by pyrophosphohydrolysis of nucleoside triphosphates (Terkeltaub, 2001). PPi regulates certain intracellular functions, but it is best recognized as a major extracellular inhibitor of tissue calcification, bone mineralization, and bone resorption (Terkeltaub, 2001). The precise mechanism by which Ank controls extracellular PPi levels is unclear. It may function directly as a PPi channel; alternatively, it could regulate the movement of PPi through another channel or it could somehow influence nucleoside triphosphate release into the extracellular milieu (Ryan, 2001).

Ank mRNA expression has been detected in the mouse at sites of both intramembranous and endochondral bone development, in tendon cells, and in the superficial layer of articular cartilage (Ho et al, 2000; Sohn et al, 2002), consistent with the proposal that Ank has an important role in the skeletal system. In addition, Ank mRNA is also expressed at relatively 
high levels in the heart and brain (Guo et al, 2002; Ho et al, 2000), but Ank function in these two complex tissue types is unknown. In this report, we demonstrate that Ank is preferentially expressed in areas of the central nervous system (CNS) with a high epileptogenic potential and significant vulnerability to seizure-induced cell death. In addition, we find a significant increase in Ank immunoreactivity throughout the limbic system in a rat seizure model. These observations suggest that Ank may function in the brain to regulate ATP and/or PPi transport into the extracellular environment and thereby modulate the membrane hyperexcitability and cell death associated with seizure activity.

\section{Results and Discussion}

\section{Characterization of Anti-Ank Antibodies and Analysis of Ank Expression in Normal Human Brain}

Previous Northern blot hybridization experiments detected Ank mRNA expression in murine (Guo et al, 2002; Ho et al, 2000) and human (Guo et al, 2002) brain, but these studies did not determine the precise cellular sites of Ank gene expression. We examined Ank expression and distribution in the human and rat (see below) brain by immunohistochemistry. First, goat anti-Ank polyclonal antiserum was obtained using two synthetic peptides as the antigen. Together these peptides corresponded to the human Ank C-terminal 37 amino acid residues, a region with $100 \%$ sequence identity to the murine Ank protein (Ho et al, 2000; Nurnberg et al, 2001). Anti-Ank antibodies were purified and concentrated by passing the serum over an antigen column. Second, the specificity of these antibodies was tested by Western blot analysis using lysates prepared from either untransfected human embryonic kidney 293 cells or 293 cells transiently transfected with an expression plasmid encoding murine Ank with an $\mathrm{N}$-terminal hexameric c-myc epitope tag. We found that the Ank-myc fusion protein migrated in a diffuse manner, but the predominant immunoreactive protein migrated with an apparent molecular mass of $\sim 75 \mathrm{kDa}$, as determined by Western blot analysis using an anti-myc epitope mAb (Fig. 1, left panel). The goat anti-Ank antibodies recognized a single protein of the same apparent molecular mass (Fig. 1, middle panel), and this immunoreactive protein was not detected when we used control goat IgG instead of anti-Ank antibodies as the primary immunologic reagent (Fig. 1, right panel). This analysis demonstrates that the goat anti-Ank antibodies specifically recognize the Ank protein. Third, immunohistochemical staining was performed to examine the Ank expression pattern in normal human brain. Ank immunoreactivity was detected primarily in neurons, with a relatively high level of Ank expression found in the thalamus, mainly in the mediodorsal and lateral dorsal nuclei (data not shown), the III and V cortical layers (external and internal pyramidal layers, respectively) (Fig. 2A), the Purkinje cells of the cerebellum (Fig. 2B), cell clusters in the dorsal portion of the pons

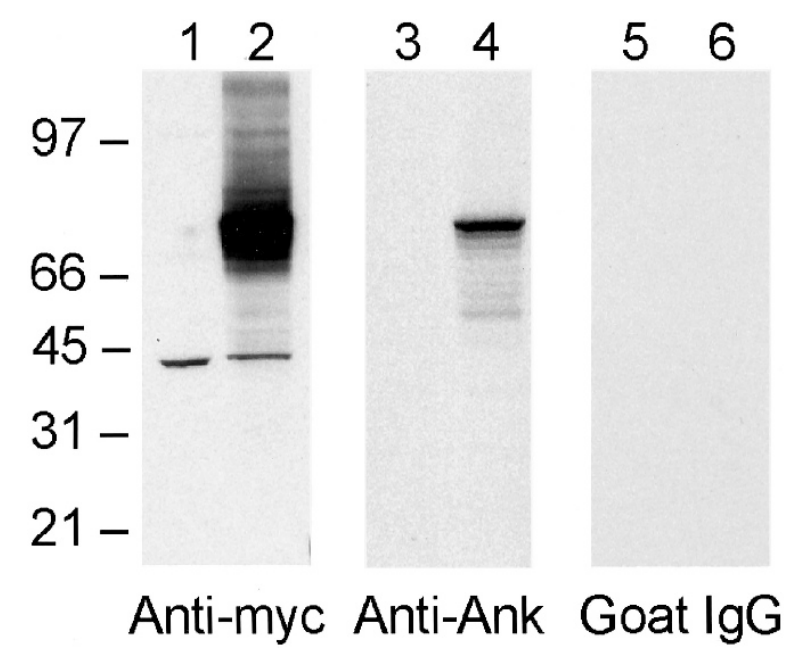

Figure 1.

Characterization of the goat anti-Ank polyclonal antiserum. Cell lysates were prepared from untransfected (lanes 1,3 , and 5) or pTracer/Ank-myctransfected (lanes 2, 4, and 6) human 293 cells. Equivalent amounts of protein were subjected to SDS-PAGE and Western blot analysis using either a mouse anti-myc epitope antibody, goat anti-Ank antibodies, or control goat IgG. Molecular masses of protein size standards (in $\mathrm{kDa}$ ) are shown on the left.

(nucleus reticularis pontis oralis, pontine nuclei, and superior olivary nucleus) and midbrain (nucleus of posterior commissure) (Fig. 2C), and neurons of the anterior horn of the spinal cord (Fig. 2D). No immunoreactivity was observed when brain sections were stained with an equivalent dilution of goat preimmune serum (not shown). These results indicate that Ank is normally expressed in areas of the CNS with high cellular metabolic activity, consistent with the postulated role for this protein in the regulation of intracellular and extracellular PPi levels.

\section{Ank Localization in Primary Neuronal Cultures}

Ank expression was investigated in cells isolated from the mouse cortex by indirect immunofluorescence analysis. Ank was not detected in glia cells, which were identified by their staining to $\beta$-isolectin (not shown). Ank expression was detected in neurons, primarily in the cell body and in some of the cell extensions, in colocalization experiments using antibodies recognizing neuron-specific nuclear protein (NeuN) (Fig. 3A). To further characterize the distribution of Ank in these neuronal extensions, Ank colocalization experiments were also conducted using antibodies directed against either an axon-specific protein (growth-associated protein-43) or a dendrite-specific protein (microtubule-associated protein-2). Although some Ank expression was detected in axons (Fig. 3B), most of the Ank immunoreactivity was found in dendrites (Fig. 3C). These results suggest that Ank may be involved in synaptic transmission and/or modulation of electrical activity because most of the excitatory inputs a neuron receives are delivered to the dendrites and most dendrites modulate the electrical state of the neuron (Harris, 1999; Harris and Kater, 1994). 

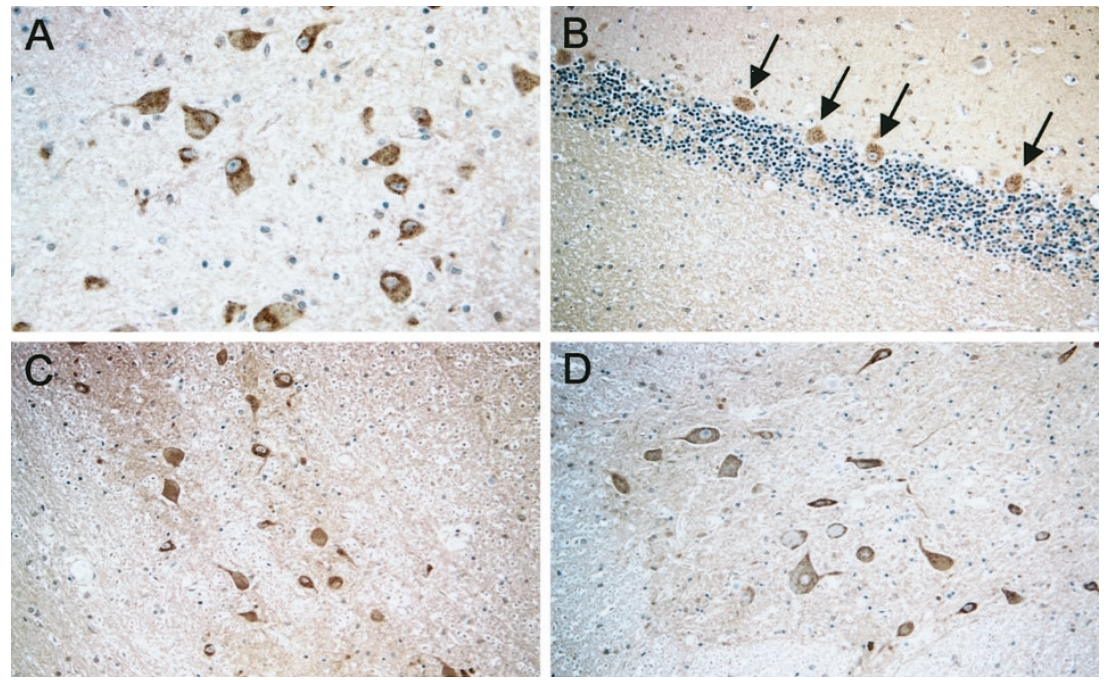

Figure 2.

Analysis of Ank expression in normal human brain. A, Neurons in the III layer of the cerebral cortex show strong staining for Ank (magnification, $\times 60$ ). B, Purkinje cells (arrows) display the most intense staining for Ank within the cerebellum (magnification, $\times 40$ ). C, Clusters of neurons in the brainstem are positive for Ank (magnification, $\times 40$ ). D, Strong staining of large neurons in the anterior horn of the spinal cord (magnification, $\times 60$ ).

\section{Ank mRNA Expression in Normal Rat Brain}

We next examined Ank gene expression in various regions of the rat brain by Northern blot hybridization analysis. Ank mRNA was present in each of the 12 regions examined, with the highest level of expression detected in thalamus, midbrain, and spinal cord (Fig. 4). It is known that the thalamus provides a powerful excitatory input to the cerebral cortex important for the generation and maintenance of seizure activity. Likewise, the generation of seizure activity reflects centrifugal propagation not only to the contralateral cortex through the thalamus and corpus callosum but also to the brainstem nuclei and to motor neurons in the spinal cord (Blume, 1992). Our observation that these are precisely the parts of the CNS where the highest expression of Ank is found suggests that this protein may play a role in the genesis and/or modulation of the abnormal electrical activity characteristic of a seizure.

\section{Seizures Increase Ank Immunoreactivity Throughout the Rat Limbic System}

The finding that most of the Ank immunoreactivity was detected in areas of the brain that are important for the generation and maintenance of seizure activity and are highly vulnerable to seizure-induced cell death (Meldrum and Corsellis, 1992) indicates that Ank may play a role in these processes. Therefore, immunohistochemical staining of rat brain sections was performed to study Ank expression and distribution in the CNS before and after chemical induction of seizure activity. In the normal rat brain, Ank immunoreactivity was detected primarily in neurons, as was observed in the normal human brain. Relatively high levels of Ank expression were observed primarily in the CA-3 layer of the hippocampus (Fig. 5A), in some scattered cells in the III and V cortical layers (Fig. 5E), in the amygdala (basolateral nuclear group) (Fig. 5G), in the pontine reticular and parapyramidal nuclei in the brainstem (not shown), and in the Purkinje cells of the cerebellum (not shown). These findings are consistent with the human brain immunohistochemistry data described earlier.

The limbic system interconnects a group of nuclei within the CNS including the amygdala and the hippocampus. The injection of kainic acid (KA) into the amygdala induces the onset of electrographic seizures, initially into the amygdala and minutes later in the hippocampus (Yepes et al, 2002). We observed a significant increase in Ank immunoreactivity in the CA-2 and CA-3 hippocampal layers (Fig. 5, B and D), in the II, III, IV, and V cortical layers (Fig. 5F), and within the amygdala (Fig. $5 \mathrm{H}$ ) at 1 hour after the onset of KA-induced seizures. These areas have been recognized previously as the most vulnerable areas of the brain after a seizure.

Northern blot hybridization analysis was then conducted using RNA isolated from the dorsal hippocampus of two control and two KA-injected (1 hour) rats to examine whether the increase in Ank immunoreactivity noted after seizure induction was a result of an increase in Ank mRNA expression. We found that Ank mRNA levels were not increased after KA injection (not shown); therefore, the increase in Ank immunoreactivity is likely not a result of an increase in Ank gene expression. This is not totally surprising considering that the KA treatment period is only 1 hour; nevertheless, we have not ruled out the possibility that KA promotes an increase in Ank mRNA translation or Ank protein stability. There are at least three other explanations for why we observed an increase in Ank immunoreactivity in the KA-injected animals. First, seizure activity could promote the redistribution or clustering of the Ank channel in such a manner that antibody recognition is increased. Second, seizure 

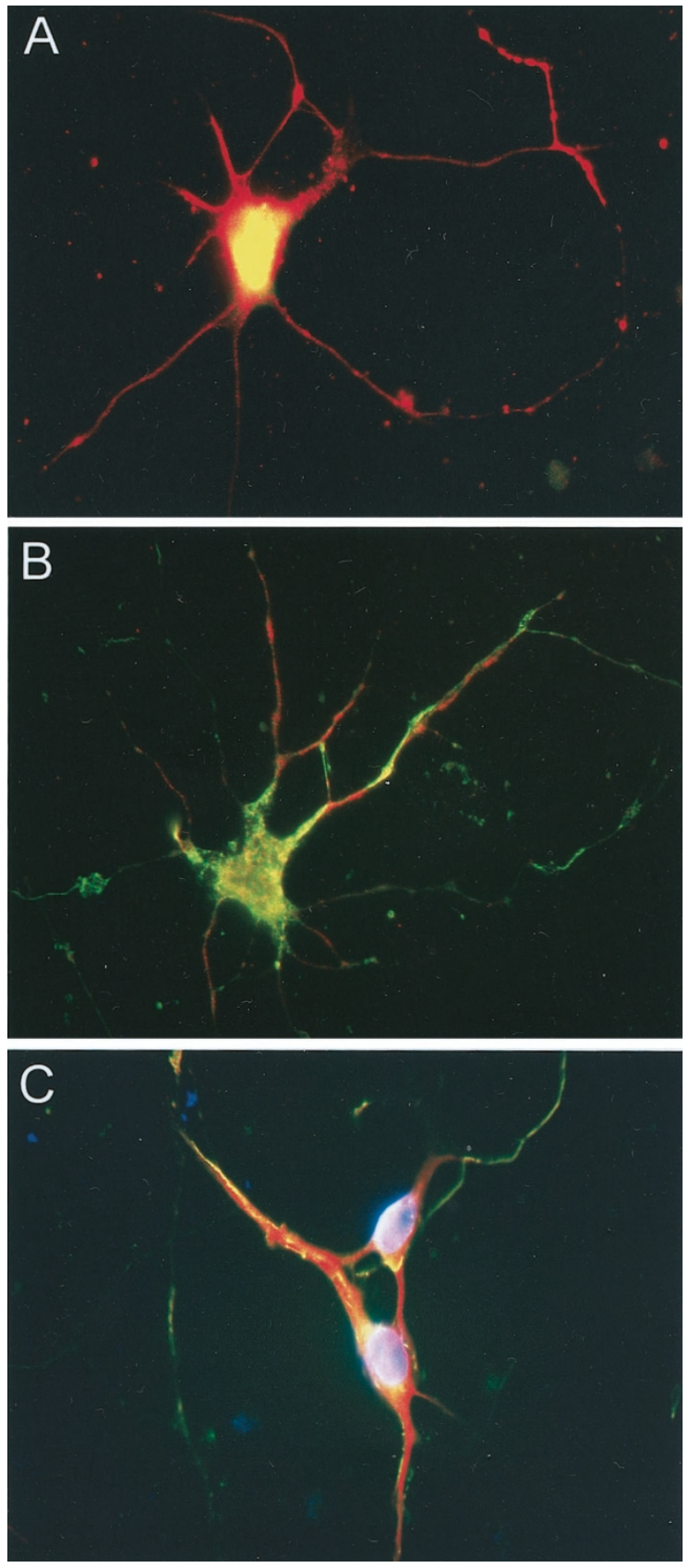

Figure 3.

Analysis of Ank distribution in mouse primary neuronal cultures. A, Cells were incubated with antibodies directed against Ank (red) in combination with antibodies recognizing the neuronal marker NeuN (green). Ank was found distributed along neuronal extensions and in the cell body where it colocalized with NeuN (yellow; magnification, $\times 100$ ). B, Cells were incubated with antibodies directed against Ank (green) in combination with antibodies recognizing the axonal marker growth-associated protein-43 (red). Ank is observed mainly in the cell body, and there is a minimal scattered localization along the axon (yellow; magnification, $\times 100$ ). C, Cells were stained with antibodies directed against Ank (green) in combination with antibodies recognizing the dendritic marker microtubule-associated protein-2 (red). The cell nuclei were stained with 4,6-diamidino-2-phenylindole (blue). Ank is found primarily in dendritic extensions (yellow; magnification, $\times 100$ ).

activity could promote a conformational change in Ank that enhances antibody recognition. Third, the antibody used for immunohistochemical staining was generated against the $\mathrm{C}$-terminal 37 amino acid resi- $\begin{array}{llllllllllll}1 & 2 & 3 & 4 & 5 & 6 & 7 & 8 & 9 & 10 & 11 & 12\end{array}$

$9.5-$

$7.5-$

$4.4-$

$2.4-$

$1.4-$

\section{Figure 4.}

Analysis of Ank mRNA expression in rat brain. Northern blot containing equivalent amounts of RNA isolated from rat brain frontal cortex (lane 1), posterior cortex (lane 2), hippocampus (lane 3), entorhinal cortex (lane 4), olfactory bulb (lane 5), striatum (lane 6), thalamus (lane 7), cerebellum (lane 8), midbrain (lane 9), pons (lane 10), medulla (lane 11), and spinal cord (lane 12) was obtained and hybridization analysis was performed using a murine Ank cDNA probe. RNA size markers (in kb) are shown on the left.

dues of the Ank protein. The topologic organization of Ank in the plasma membrane has not been experimentally determined, but a model proposed by Nurnberg et al (2001) indicates that this $\mathrm{C}$-terminal region is located in the cytoplasm. Therefore, seizure activity could promote an increase in neuronal cell permeability, which would make the antibody epitope more accessible.

Ank is thought to function primarily as a channel or regulatory protein that transports $\mathrm{PPi}$ into the extracellular environment, thereby indirectly controlling the process of tissue calcification (Ho et al, 2000). However, the available data do not exclude the possibility that Ank may influence ATP export (Ryan, 2001). A seizure is a paroxysmal and synchronous discharge of a group of neurons and is associated with a significant increase in the brain metabolic rate (Chapman et al, 1977; Heinemann et al, 2002). In this situation, the regulation of intracellular and extracellular ATP, PPi, and calcium concentrations is likely to be critical. In neurons, ATP is stored in synaptic vesicles and released from terminals in a calcium-dependent manner. Once in the synaptic space, ATP binds to extracellular receptors where it has an excitatory effect, and it is rapidly removed from the synaptic cleft by enzymatic action. Furthermore, during a seizure there is a decrease in the extracellular concentration of calcium that makes the membrane more excitable (Coulter, 1999; Meldrum, 2002; Somjen et al, 1986). Indeed, excessive entry of calcium into the cell is thought to be one of the key events in seizure-induced cell death (Meldrum, 2002). We propose that the Ank protein expressed in the brain may play a role in both ATP and PPi release into the extracellular environment. The increase in ATP levels would promote the neuronal hyperexcitability characteristic of a seizure, while the increase in PPi levels would influence intracellular and extracellular calcium concentrations, important factors for the development of increased membrane excitability and cell death, respectively. 


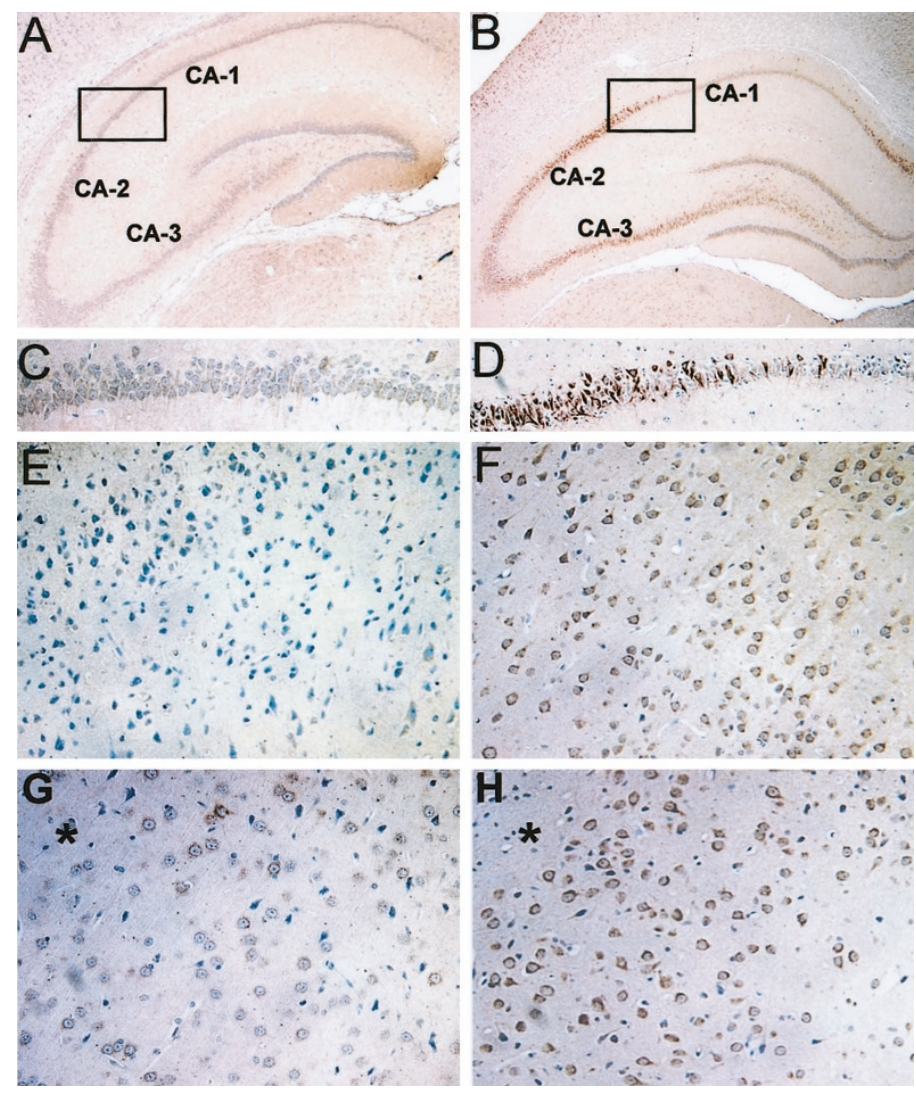

Figure 5.

Analysis of Ank expression in rat hippocampus, cortex, and amygdala after KA injection. A, C, E, and G, Ank expression in normal hippocampus (A and C), cortex $(E)$, and amygdala $(G)$ in an animal without seizures. $B, D, F$, and $H$, Ank expression in hippocampus (B and D), cortex (F), and amygdala (H) 1 hour after the onset of seizures. B, Significant increase in Ank immunoreactivity in the CA-2 and CA-3 hippocampal layers after a seizure. A and B (Insets), location where higher magnification photographs were taken, which are shown in $C$ and D. C, Scattered cells positive for Ank. D, It is evident that after a seizure there is a significant increase in Ank immunoreactivity in the CA-2 hippocampal layer (left side) compared with the CA-1 layer (right side). E and G, In a normal animal there are scattered neurons positive for Ank staining in the III layer of the cerebral cortex and in the amygdala, respectively. F and H, Significant increase in Ank immunoreactivity in cerebral cortex $(\mathrm{F})$ and amygdala $(\mathrm{H})$ after a seizure (magnification: $\times 20$ for $\mathrm{A}$ and $\mathrm{B}, \times 40$ for $\mathrm{C}-\mathrm{H}$ ). $\mathrm{G}$ and $\mathrm{H}$, Boundaries of the basolateral amygdala complex are indicated with asterisks.

\section{Materials and Methods}

\section{Generation and Purification of Anti-Ank Serum}

Ank peptides were synthesized on an octa-branched core matrix using the multiple antigen peptide approach described by Tam (1988). Briefly, the peptides KKKMENESATEGEDSAMTD and MPPTEEVTDIVEMREENE, which together represent residues 457 to 493 of both human and murine Ank (Ho et al, 2000), were each synthesized on Fmoc (fluoren-9ylmethoxycarbonyl)-multiple antigen peptide eightbranch resin (Applied Biosystems, Foster City, California), cleaved from the resin with trifluoroacetate, and then purified by reverse-phase HPLC. Antibody production services were provided by Cocalico Biologicals (Reamstown, Pennsylvania). Briefly, a goat was first injected with $0.5 \mathrm{mg}$ of the antigen in complete Freund's adjuvant (Calbiochem, La Jolla, California) and then boosted nine times with $0.25 \mathrm{mg}$ of the antigen in incomplete adjuvant (Calbiochem). For affinity purification of the antiserum, the peptides were attached to $\mathrm{CNBr}$-activated Sepharose 4B resin (Amersham Biosciences, Piscataway, New Jersey) according to the manufacturer's instructions. Serum was applied to the column, and the resin was then washed one time each with PBS, $0.1 \mathrm{M} \mathrm{NaHCO}_{3}, 0.1 \mathrm{M}$ $\mathrm{NaHCO}_{3} / 0.5 \mathrm{M} \mathrm{NaCl}$, and again with PBS. Bound antibody was eluted using $0.2 \mathrm{M}$ glycine $\mathrm{pH} 2.5$, and each fraction was neutralized and then analyzed by SDS-PAGE. Appropriate fractions were pooled, dialyzed, and filter sterilized.

\section{Construction of the Ank Expression Plasmid}

The plasmid pTracer/Ank-myc was constructed in three steps. First, PCR assays were performed to generate a single cDNA clone from the two partial cDNAs described in our previous report (Guo et al, 2002). One of the sense oligonucleotide primers, primer 1 (see below), contained several base mutations to introduce an optimal Ank mRNA translation start site; in addition, the PCR assays were designed so that the resulting cDNA encoded the entire Ank open-reading frame but did not contain DNA sequence encoding the relatively long $(\sim 1.2 \mathrm{~kb})$ Ank mRNA 3'-untranslated region. All of the PCR assays were performed using Vent DNA Polymerase (New England Biolabs, Beverly, Massachusetts) and oligo- 
nucleotide primers obtained from IDT Inc. (Coralville, lowa). In one reaction, the plasmid pCRII/Ank (Guo et al, 2002) was used as the template in combination with the sense mutagenic primer 5'ACGGCCGCCACCATGGTGAAATTCCCGG-3' (primer 1) representing Ank nucleotides 18 to 46 (GenBank accession number AF001532) and an antisense primer complementary to Ank nucleotides 97 to 120 (primer 2, GenBank AF001533). In a second reaction, the plasmid pBluescript/mAnk (Guo et al, 2002) was used as the template in combination with a sense primer representing Ank nucleotides 1 to 24 (primer 3, GenBank AF001533) and an antisense primer complementary to Ank nucleotides 1125 to 1152 (primer 4, GenBank AF001533). The PCR products obtained in these two reactions were isolated and then combined to serve as templates for a third reaction using primers 1 and 4 described above. The DNA product was isolated, ligated into the vector pCR-Script using a PCR fragment cloning kit (Stratagene, La Jolla, California), and then sequenced to confirm that no mutations were introduced during this series of PCR assays. Second, a DNA fragment containing a hexameric repeat of the Myc epitope tag was isolated by ClalEcoRI digestion of the plasmid pCS2 + MT (a kind gift of R. Friesel, Maine Medical Center Research Institute) and then ligated into the pCR-Script/Ank plasmid digested with the same two restriction enzymes. This step placed an ATG start site and multi-myc tag at the $\mathrm{N}$-terminus of Ank. The identity of this construct was verified by DNA sequence analysis. Finally, in the third step, the pCR-Script/Ank-myc plasmid was digested with Kpnl-Notl to release the insert. This DNA fragment was isolated and then ligated into the vector pTracer-CMV (Invitrogen, Carlsbad, California) that had been digested with the same two restriction enzymes. The resulting plasmid, pTracer/Ank-myc, was purified from transformed bacterial cells using a Plasmid Maxi-kit (Qiagen, Valencia, California) according to the manufacturer's instructions.

\section{Plasmid Transfection and Western Blot Analysis}

Human embryonic kidney 293 cells (American Type Culture Collection, Manassas, Virginia) were grown in Eagle's minimum essential medium (BioWhittaker, Walkersville, Maryland), $1 \mathrm{~mm}$ sodium pyruvate (BioWhittaker), $1 \times$ nonessential amino acids (Mediatech, Herndon, Virginia), and $10 \%$ fetal bovine serum (Hyclone, Logan, Utah). They were transfected with the pTracer/Ank-myc plasmid using Lipofectamine Plus (Invitrogen) according to the manufacturer's instructions. At 48 hours after transfection, the cells were harvested and lysed in HNTG buffer (20 mM HEPES, $150 \mathrm{~mm} \mathrm{NaCl}, 1 \%$ Triton X-100, 10\% glycerol, $1.5 \mathrm{~nm}$ $\mathrm{MgCl}_{2}, 1 \mathrm{~mm}$ EGTA). Untransfected cells were also harvested and lysed in an identical manner. Protein concentrations were determined using the BCA protein assay kit (Pierce, Rockford, Illinois). Equivalent amounts of each cell lysate were then subjected to SDS-PAGE using a $4 \%$ to $12 \%$ Bis-Tris NuPage gel (Invitrogen) without heat denaturation of the samples.
Proteins were transferred to nitrocellulose membranes (Schleicher and Schuell, Keene, New Hampshire) and then visualized using Ponceau S Stain (Sigma, St. Louis, Missouri). The three membranes were blocked for 1 hour at $37^{\circ} \mathrm{C}$ in TBST $(25 \mathrm{~mm}$ Tris/ $\mathrm{HCl}, \mathrm{pH} 7.5$, $150 \mathrm{~mm} \mathrm{NaCl}, 0.1 \%$ Tween-20) containing 5\% nonfat dry milk. One membrane was incubated for 1 hour at room temperature in TBST containing 5\% BSA and a 1:500 dilution of anti-myc mAb 9E10 (a kind gift of S. Robinson, Holland Lab), washed three times with TBST, and incubated for 1 hour in TBST containing $5 \%$ nonfat dry milk and a 1:5000 dilution of horseradish peroxidase-conjugated goat anti-mouse IgG (Santa Cruz, Santa Cruz, California). The other two membranes were incubated for 1 hour in TBST containing $5 \%$ nonfat dry milk and a 1:500 dilution of either goat anti-Ank IgG or control goat IgG (Sigma), washed as above, and then incubated for 1 hour in TBST containing 5\% nonfat dry milk and a 1:10,000 dilution of horseradish peroxidase-conjugated donkey anti-goat IgG (Santa Cruz). For all Western blots, membranes were washed three times in TBST and bound secondary antibodies were detected using ECL Western Blotting Detection Reagents (Amersham Pharmacia, Piscataway, New Jersey).

\section{Mouse Neuronal Cell Culture and Immunofluorescence}

Primary neuronal cultures of mouse cortex were prepared from embryonic Day 15 CD1 mice as described elsewhere (Bacskai et al, 2000). Briefly, the cortices were isolated in $\mathrm{Ca}^{2+}$-free PBS and homogenized in neurobasal medium containing $10 \%$ fetal bovine serum (Invitrogen), $2 \mathrm{~mm}$ glutamine, $100 \mathrm{U} / \mathrm{ml}$ penicillin, and $100 \mathrm{U} / \mathrm{ml}$ streptomycin. The cells were plated onto polylysine-coated coverslips in 12-well tissue culture plates. After 60 minutes at $37^{\circ} \mathrm{C}$, the medium was removed and attached cells were incubated in neurobasal medium containing $1 \times$ B27 supplement (Invitrogen). One week later the cells were washed twice with PBS, fixed in 3\% formaldehyde in PBS, and then permeabilized and blocked in $0.1 \%$ saponin and $5 \%$ BSA in PBS for 1 hour. All antibodies were diluted in the above blocking buffer, and coverslips were washed three times in between each step. Cells were incubated with goat anti-Ank antibodies (1:1000) in combination with either anti-NeuN mAb (1:500; Chemicon, Temecula, California), anti-growthassociated protein-43 polyclonal antibody (1:500; Chemicon), or anti-microtubule-associated protein-2 mAb (1:500; Sigma) for 1 hour at room temperature. Donkey anti-goat, anti-rabbit, or anti-mouse secondary antibodies conjugated to either FITC or Rhodamine Red-X (Jackson ImmunoResearch, West Grove, Pennsylvania) were diluted to 1:500 and applied for 1 hour at room temperature. Cells were stained with anti-Ank antibodies in combination with FITC-labeled $\beta$-isolectin (1:500; Sigma) to examine Ank expression in glia. In some cases the cells were counterstained with 4,6-diamidino-2-phenylindole (Molecular Probes, Eugene, Oregon) for 2 to 3 seconds. Coverslips were 
rinsed with PBS and mounted on glass slides with Gel/Mount (Biomedia, Foster City, California).

\section{Northern Blot Hybridization Analysis}

A blot containing $20 \mu \mathrm{g}$ of total RNA isolated from various regions of rat brain was obtained from OriGene Technologies (Rockville, Maryland). Membrane prehybridization, hybridization, and washing conditions were as described (Meighan-Mantha et al, 1999). The cDNA hybridization probe was an $\sim 2.2-\mathrm{kb}$ EcoRIXhol fragment of pBluescript/mAnk (Guo et al, 2002).

\section{Animal Preparation and Surgery}

Adult Sprague-Dawley rats weighing 350 to $400 \mathrm{~g}$ were used. Animals ( $n=3$ per group) were anesthetized and placed in a stereotaxic apparatus; $3 \mu \mathrm{l}$ of 1.0 nм KA (A.G. Scientific, San Diego, California) in PBS, or PBS alone, was injected unilaterally into the amygdala. The coordinates of the KA injection were bregma, $-3.60 \mathrm{~mm}$; medial-lateral, $5 \mathrm{~mm}$; and dorso-ventral, $9 \mathrm{~mm}$. Injections were given during 60 seconds, and the needle was left in place for another 3 minutes to prevent reflux. After KA injection, the animals experienced convulsive seizures characterized by myoclonic jerks involving the head and neck, followed by unilateral tonic-clonic activity in the limbs and finally generalized bilateral convulsive activity, as previously described (Yepes et al, 2002).

\section{Immunohistochemistry}

Human brain sections were obtained from Novagen (Madison, Wisconsin). This tissue, which came from a 26-year-old male whose brain was determined to be normal by a pathologist, was fixed in $4 \%$ paraformaldehyde, embedded in paraffin, and sectioned at 5-um thickness by Novagen. Rats underwent transcardiac perfusion 60 minutes after the onset of seizures. The brains were then removed, immediately frozen in $\mathrm{OCT}$, and stored at $-70^{\circ} \mathrm{C}$. Cryostat sections $(0.5 \mu \mathrm{m})$ were then processed for Ank detection. The sections were deparaffinized and immersed in 100\% methanol/0.3\% $\mathrm{H}_{2} \mathrm{O}_{2}$ for 30 minutes to exhaust endogenous peroxidase activity, and then preincubated with $10 \%$ rabbit serum followed by goat anti-Ank antibodies at a 1:1000 dilution for 1 hour at room temperature. After a wash with PBS, a 1:200 dilution of biotinylated anti-goat secondary antibody (Vector Laboratories, Burlingame, California) was added for 30 minutes. The sections were then washed in PBS, incubated with a 1:100 dilution of $A B C$ Elite reagent (Vector Laboratories), developed with 3.3'-diaminobenzidine for 4 minutes, and counterstained with Mayer's hematoxylin for 2 minutes. The Ank staining pattern was reproducible between animals, and representative immunohistochemistry data are shown in each figure.

\section{Acknowledgements}

We thank Ms. Sheau-Line Feng and Dr. Patrick Donohue for technical assistance, Ms. Ewa Maddox for peptide synthesis and purification, Ms. Sue Robinson for providing the anti-myc mAb, Dr. Bob Friesel for providing the $\mathrm{pCS}+\mathrm{MT}$ plasmid, and Ms. Bonnie Van Veldhuizen for excellent secretarial assistance.

\section{References}

Bacskai BJ, Xia MQ, Strickland DK, Rebeck GW, and Hyman BT (2000). The endocytic receptor protein LRP also mediates neuronal calcium signaling via $\mathrm{N}$-methyl-D-aspartate receptors. Proc Natl Acad Sci USA 97:11551-11556.

Blume W (1992). Motor system: Anatomy, physiology and epileptogenesis. In: Willye E, editor. The treatment of epilepsy. Baltimore: Williams and Wilkins, 82-92.

Chapman AG, Meldrum BS, and Siesjo BK (1977). Cerebral metabolic changes during prolonged epileptic seizures in rats. J Neurochem 28:1025-1035.

Coulter DA (1999). Chronic epileptogenic cellular alterations in the limbic system after status epilepticus. Epilepsia 40(Suppl 1):S23-S33.

Guo Y, Hsu DKW, Feng SY, Richards CM, and Winkles JA (2002). Polypeptide growth factors and phorbol ester induce progressive ankylosis (ank) gene expression in murine and human fibroblasts. J Cell Biochem 84:27-38.

Harris KM (1999). Structure, development, and plasticity of dendritic spines. Curr Opin Neurobiol 9:343-348.

Harris KM and Kater SB (1994). Dendritic spines: Cellular specializations imparting both stability and flexibility to synaptic function. Annu Rev Neurosci 17:341-371.

Heinemann U, Buchheim K, Gabriel S, Kann O, Kovacs R, and Schuchmann S (2002). Cell death and metabolic activity during epileptiform discharges and status epilepticus in the hippocampus. Prog Brain Res 135:197-210.

Ho AM, Johnson MD, and Kingsley DM (2000). Role of the mouse ank gene in control of tissue calcification and arthritis. Science 289:265-270.

Meighan-Mantha RL, Hsu DKW, Guo Y, Brown SAN, Feng SY, Peifley KA, Alberts GF, Copeland NG, Gilbert DJ, Jenkins NA, Richards CM, and Winkles JA (1999). The mitogeninducible Fn14 gene encodes a type I transmembrane protein that modulates fibroblast adhesion and migration. J Biol Chem 274:33166-33176.

Meldrum BS (2002). Concept of activity-induced cell death in epilepsy: Historical and contemporary perspectives. Prog Brain Res 135:3-11.

Meldrum BS and Corsellis JAN (1992). Epilepsy. In: Adams $\mathrm{JH}$, Corsellis JAN, and Duchen LW, editors. Greenfield's neuropathology. New York: John Wiley and Sons, 921-950.

Nociti FH Jr, Berry JE, Foster BL, Gurley KA, Kingsley DM, Takata T, Miyauchi M, and Somerman MJ (2002). Cementum: A phosphate-sensitive tissue. J Dent Res 81:817-821.

Nurnberg P, Thiele H, Chandler D, Hohne W, Cunningham ML, Ritter H, Leschik G, Uhlmann K, Mischung C, Harrop K, Goldblatt J, Borochowitz ZU, Kotzot D, Westermann F, Mundlos S, Braun HS, Laing N, and Tinschert S (2001). Heterozygous mutations in ANKH, the human ortholog of the 
mouse progressive ankylosis gene, result in craniometaphyseal dysplasia. Nat Genet 28:37-41.

Pendleton A, Johnson MD, Hughes A, Gurley KA, Ho AM, Doherty M, Dixey J, Gillet P, Loeuille D, McGrath R, Reginato A, Shiang R, Wright G, Netter P, Williams C, and Kingsley DM (2002). Mutations in ANKH cause chondrocalcinosis. Am J Hum Genet 71:933-940.

Reichenberger E, Tiziani V, Watanabe S, Park L, Ueki Y, Santanna C, Baur ST, Shiang R, Grange DK, Beighton P, Gardner J, Hamersma H, Sellars S, Ramesar R, Lidral AC, Sommer A, Raposo do Amaral CM, Gorlin RJ, Mulliken JB, and Olsen BR (2001). Autosomal dominant craniometaphyseal dysplasia is caused by mutations in the transmembrane protein ANK. Am J Hum Genet 68:1321-1326.

Russell RG (1976). Metabolism of inorganic pyrophosphate (PPi). Arthritis Rheum 19(Suppl 3):465-478.

Ryan LM (2001). The ank gene story. Arthritis Res 3:77-79.

Sohn P, Crowley M, Slattery E, and Serra R (2002). Developmental and TGF-beta-mediated regulation of Ank mRNA expression in cartilage and bone. Osteoarthritis Cartilage 10:482-490.
Somjen GG, Aitken PG, Giacchino JL, and Mcnamara JO (1986). Interstitial ion concentrations and paroxysmal discharges in hippocampal formation and spinal cord. Adv Neurol 44:663-680.

Tam JP (1988). Synthetic peptide vaccine design: Synthesis and properties of a high-density multiple antigenic peptide system. Proc Natl Acad Sci USA 85:5409-5413.

Terkeltaub RA (2001). Inorganic pyrophosphate generation and disposition in pathophysiology. Am J Physiol Cell Physiol 281:C1-C11.

Yepes M, Sandkvist M, Coleman TA, Moore E, Wu JY, Mitola D, Bugge TH, and Lawrence DA (2002). Regulation of seizure spreading by neuroserpin and tissue-type plasminogen activator is plasminogen-independent. J Clin Invest 109:15711578. 